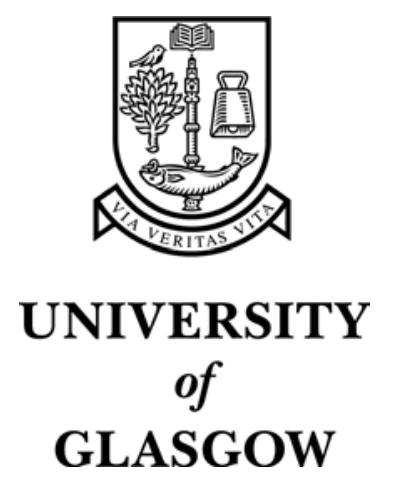

Gawthrop, P.J. and Wang, L. (2005) Data compression for estimation of the physical parameters of stable and unstable linear systems. Automatica 41(8):1313-1321.

http://eprints.gla.ac.uk/archive/00001966/ 


\title{
Data Compression for Estimation of the Physical Parameters of Stable and Unstable Linear Systems
}

\author{
Peter J Gawthrop ${ }^{\mathrm{a}, 1}$ Liuping Wang ${ }^{\mathrm{b}}$ \\ ${ }^{a}$ Centre for Systems and Control and Department of Mechanical Engineering, University of Glasgow, GLASGOW. G12 8QQ Scotland. \\ P.Gawthrop@eng.gla.ac.uk \\ ${ }^{\mathrm{b}}$ Discipline of Electrical Energy and Control Systems, School of Electrical and Computer Engineering, RMIT University, Melbourne, \\ Victoria 3000, Australia
}

\begin{abstract}
A two-stage method for the identification of physical system parameters from experimental data is presented. The first stage compresses the data as an empirical model which encapsulates the data content at frequencies of interest. The second stage then uses data extracted from the empirical model of the first stage within a non-linear estimation scheme to estimate the unknown physical parameters. Furthermore, the paper proposes use of exponential data weighting in the identification of partially unknown, unstable systems so that they can be treated in the same framework as stable systems. Experimental data are used to demonstrate the efficacy of the proposed approach.
\end{abstract}

Key words: Parameter estimation; partially known systems; basis function.

\section{Introduction}

Many engineering systems of interest to the control engineer are partially known in the sense that the system structure, together with some system parameters are known, but some system parameters are unknown. This gives rise to a problem of parameter estimation when values for the unknown parameters are to be determined from experimental data comprising measurements of system inputs and outputs. There is a considerable literature in the area (An, Atkeson, and Hollerbach, 1988; Canudas de Wit, 1988; Dasgupta, Anderson, and Kaye, 1986; Gawthrop, Jones, and MacKenzie, 1992; Gawthrop, 2000a,b; Nagy and Ljung, 1991). Although, in special cases, such identification may be linear-in-the parameters (An et al., 1988) or polynomial-in-the parameters (Gawthrop et al., 1992), in general the problem is nonlinearin-the parameters. This means that, in general, the resultant optimisation problem is not quadratic or polynomial, and may even be non-convex. In such cases, the optimisation task is eased by knowing (rather than deducing numerically) the derivative of the error function with respect to the unknown system parameters. The generation of such sensitivity information is aided by the symbolic methods for nonlinear systems modelling, analysis and optimisation which are currently strong research areas (Munro, 1999) driven by the

\footnotetext{
1 Corresponding author
}

ready availability of symbolic computational tools. In particular, the bond graph approach (Gawthrop and Smith, 1996; Karnopp, Margolis, and Rosenberg, 1990; Ljung and Glad, 1994) has been used to generate models both applicable to control design (Gawthrop, 1995; Gawthrop and Ronco, 2000) and partially-known system identification (Gawthrop, 2000a, 2003; Nagy and Ljung, 1991). Bond graph models are used in all the examples of this paper, but are not discussed further here.

Data acquisition systems typically yield large amounts of discrete-time data. On the other hand, the aforementioned partially known systems are usually best expressed in continuous-time differential equation form and, even with these sensitivity function enhancements, use of the raw data may lead to unacceptable computational times. Thus, although it is, in principle, possible to use algorithms for partially-known system identification directly on the raw data, it is not practically useful. In addition, the raw data may contain complex system disturbance information which may require a sophisticated optimisation algorithm to achieve desirable results.

In this paper, the authors propose a two-stage identification procedure to extract physical parameters from discrete-time data pertaining to partially known systems. The first stage (which we call data compression) analyses the raw data to obtain a parameter vector $\theta$ describing an empirical model obtained from the data. The second stage uses this empiri- 
cal model (parameterised by $\theta$ ) to generate continuous-time data suitable for identifying physical parameters. Because the first stage is essentially a linear-in the parameter problem, not only can large amounts of data be processed rapidly, but also established system identification tools can be used to obtain data-quality models (Ljung, 1999). Because the second stage uses a relatively short length of relatively noise free data, the iteration time and convergence properties are much improved compared to using the raw data directly. The continuous time step response is used as the empirical model as it has a transparent representation in terms of gain, time delay and time constant and thus it is widely accepted by engineers and practitioners. Other forms of empirical model are also possible within this context. The basic idea of a two-stage method is not new, see for example, Ljung (1999, Section 10.4) and Wang, Gawthrop, Chessari, Podsiadly, and Giles (2004); our method is new insofar as it uses the FSF approach for the first stage and a physical model-based approach for the second.

In order for the same framework to be applicable to unstable systems, this paper proposes the use of exponential data weighting in the data compression procedure. This exponential weighting converts an unstable system into a stable system with the same unknown parameters to which the two-stage approach is applicable.

The motivation for this work is to generate models suitable for model-based predictive control (Mayne et al, 2000; Rawlings, 2000), in particular models suitable for continuous time methods, such as those of Wang (2001) and Gawthrop and Ronco (2000, 2002).

The outline of the paper is as follows. Section 2 considers the frequency sampling filter approach to data compression and extends the procedure to cope with unstable systems. Section 3 considers physical parameter estimation and section 4 considers frequency-domain approaches. Section 5 gives illustrative experimental results using data obtained from both electrical and electro-mechanical systems. Section 6 concludes the paper.

\section{Data compression}

The first stage of the two-stage process is data compression: encapsulating the important features of the measured data into a few parameters within an empirical system model. There are many possible empirical models available including ARX (Ljung, 1999) and general basis-function approaches (Ninness and Gustafsson, 1997; Wahlberg, 1991; Wang and Cluett, 2000). In a fast-sampling environment, it is known that discrete-time ARX models encounter numerical ill-conditioning (Åström, Hagander, and Sternby, 1980) as the sampling rate increases; and the problem is worse for unstable systems. On the contrary, the frequency-sampling filter (FSF) approach of Bitmead and Anderson (1981); Wang and Cluett $(1997,2000)$, lies between the continuous and discrete-time domains and the coefficients converge to sampling-rate independent values. This latter approach is discussed in Section 2.1 and extended to unstable systems in Section 2.2.

\subsection{Stable systems}

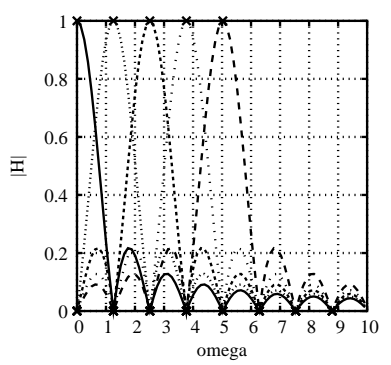

(a) Frequency responses

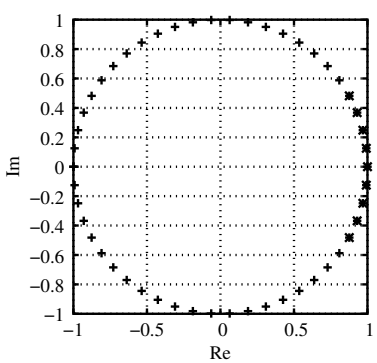

(b) Pole locations

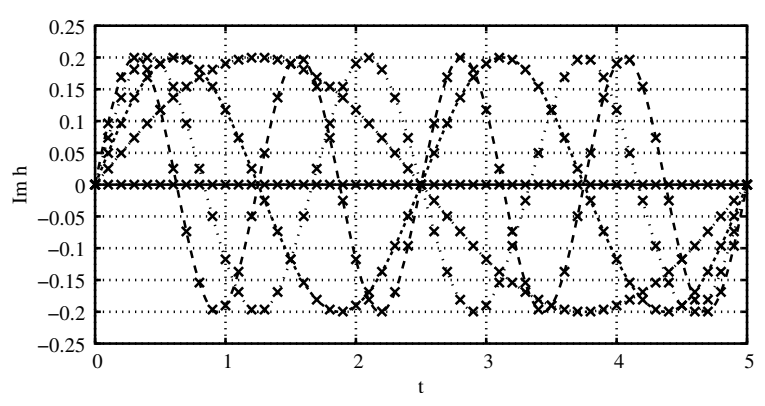

(c) Time responses

Fig. 1. Frequency-sampling filters

The book by Wang and Cluett (2000) gives a comprehensive discussion of the frequency-sampled filter approach (including its relation to the discrete Fourier transform); this section provides a brief discussion of the material required for this paper. We consider linear time-invariant continuoustime systems with output $y(t)$ and input $u(t)$ uniformly sampled with time interval $\Delta$ to give input and output sequences $y_{i}=y(i \Delta)$ and $u_{i}=u(i \Delta)$. In the time-domain, the input and output sequences are related by $y_{i}=g_{i} * u_{i}$ where $g_{i}$ is the discrete-time system impulse response and $*$ is the convolution operator. In the $z$-transform domain, $\bar{Y}(z)=\bar{G}(z) \bar{U}(z)$ where $\bar{Y}$ and $\bar{U}$ are the $z$-transforms of $y_{i}$ and $u_{i}$ respectively and $\bar{G}$ the corresponding transfer function. In this section, it is assumed that the system is stable and can be associated with a settling time $T=N \Delta$; the time after which the system impulse response is sufficiently small: $\left|g_{i}\right|<\varepsilon \forall i>N$.

The frequency-sampling filter FSF approach approximates 
the transfer function $\bar{G}(z)$ as:

$$
\begin{aligned}
\bar{G}_{f s f}(z) & =\sum_{k=-\frac{n-1}{2}}^{\frac{n-1}{2}} \theta_{k} \bar{H}_{k}(z) \\
\bar{H}_{k}(z) & =\frac{1}{N} \frac{1-z^{-N}}{1-e^{j \Omega k} z^{-1}}
\end{aligned}
$$

where $n$ is odd and the frequency sample interval $\Omega=\frac{2 \pi}{T}$ $\bar{H}_{k}(z)$ is the $k$ th frequency sampling filter (FSF) and $\theta_{k}$ the corresponding (complex) parameter. The name arises because the $k$ th FSF has a frequency response with a peak at $\omega=k \Omega$. Figure 1(a) shows the superimposed frequency responses of $\bar{H}_{k}(z)$ for $0 \leq k \leq 4$ when $T=5$ implying $\Omega=1.26$ for a frequency range $0 \leq \omega \leq 10$. The symbol " $x$ " marks the frequency samples which coincide with the peaks of the FSFs. The $k$ th filter of (2) has the discrete-time impulse response $\bar{h}_{k}(i)$

$$
\bar{h}_{k}(i)=\frac{1}{N} e^{j \Omega k i} i<N
$$

As discussed by Wang and Cluett (1997, 2000), choosing $n=N$ gives an exact match $\bar{G}_{f s f}(z)=\bar{G}(z)$. Choosing $n<N$ gives an approximate match $\bar{G}_{f s f}(z) \approx \bar{G}(z)$ for a frequency range $0 \leq \omega \leq N \Omega$. This situation is summarised in Figure 1(b) which shows $N=50$ potential FSF poles (marked by "+") equispaced around the unit circle and the $n=9$ actual FSF poles clustered around $z=1$ on the unit circle. Particularly in the context of fast (with respect to system time constants) sampling, a good approximation can be obtained with $n<<N$. The significance of this approximation lies in the fact that the neglected process frequency parameters correspond to higher frequency region of the system, which in many applications have severe noise corruption.

The FSF equation (1) can be rewritten in vector form as:

$$
\bar{G}_{f s f}(z)=\theta^{T} \bar{F}(z)
$$

where

$$
\bar{F}(z)=\left(\begin{array}{c}
\bar{H}_{0}(z) \\
\bar{H}_{-1}(z) \\
\bar{H}_{1}(z) \\
\ldots \\
\bar{H}_{-\frac{n-1}{2}}(z) \\
\bar{H}_{\frac{n-1}{2}}(z)
\end{array}\right) \quad \theta=\left(\begin{array}{c}
\theta_{0} \\
\theta_{-1} \\
\theta_{1} \\
\ldots \\
\theta_{-\frac{n-1}{2}} \\
\theta_{\frac{n-1}{2}}
\end{array}\right)
$$

In time-domain terms:

$$
y_{i}=\theta^{T} f_{i} * u_{i}
$$

where $f_{i}$ is the (discrete-time) impulse response corresponding to $\bar{F}(z)$. The convolution is, in practice performed by the usual time-domain filtering operation. Equation (4) is in the conventional linear-least squares form and so the parameter estimate $\hat{\theta}$ may be chosen to minimise a performance index of the form

$$
J(M, \hat{\theta})=\sum_{i=0}^{M}\left|e_{i}\right|^{2}
$$

where $e_{i}=y_{i}-\hat{y}_{i}$ and $\hat{y}_{i}$ is given by (6) with $\theta$ replaced by $\hat{\theta}$. Defining $Y_{M}=\left(\begin{array}{llll}y_{0} & y_{1} & \ldots & y_{M}\end{array}\right)^{T}, \phi_{i}=f_{i} * u_{i}$ and $\Phi_{M}=$ $\left(\begin{array}{llll}\phi_{0} & \phi_{1} & \ldots & \phi_{M}\end{array}\right)^{T}$ then the Least Squares estimate is

$$
\hat{\theta}=\left(\Phi_{M}^{*} \Phi_{M}\right)^{-1} \Phi_{M}^{*} Y_{M}
$$

Although the FSF approach is cast in the discrete-time domain and the corresponding $z$-transform domain, the resultant model can be used to obtain continuous-time step and frequency responses as follows(Wang and Cluett, 2000). Using $z=e^{j \omega \Delta}$, (1) and (2) can be rewritten in frequency domain form as

$$
\begin{aligned}
G(j \omega) \approx G_{f s f}(j \omega) & =\sum_{k=-\frac{n-1}{2}}^{\frac{n-1}{2}} \theta_{k} H_{k}(j \omega) \\
H_{k}(j \omega) & =\bar{H}_{k}\left(e^{j \omega \Delta}\right) \text { for } \omega<N \Omega
\end{aligned}
$$

Similarly, the system impulse response $g(t)$ can be approximately computed using the continuous-time equivalent of (3)

$$
\begin{aligned}
g(t) \approx g_{f s f}(t) & =\sum_{k=-\frac{n-1}{2}}^{\frac{n-1}{2}} \theta_{k} h_{k}(t) \\
h_{k}(t) & =\frac{1}{T} e^{j k \Omega t} \text { for } t<N \Delta
\end{aligned}
$$

And the step response $y_{s}(t)$ from:

$$
y_{s}(t)=\int_{0}^{t} g(\tau) d \tau
$$

\subsection{Unstable systems}

There are two problems associated with applying the results of Section 2.1 to unstable systems:

(1) the data itself may be theoretically unbounded or, in practice, reach unmodelled physical limits

(2) the FSF approach assumes that the system is asymptotically stable, and thus has a step response which is asymptotically constant.

In this paper, it is assumed that the first problem is solved by closed-loop identification using a stabilising controller 


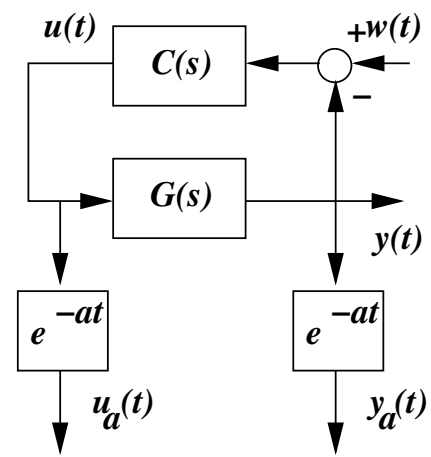

(a) Exponential weighting

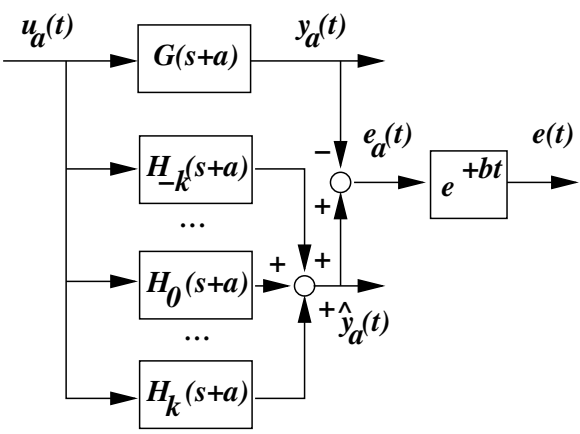

(b) Identification

Fig. 2. Unstable systems

$C(s)$ as in Figure 2(a). As discussed, for example, by Ljung (1999), identification in closed-loop gives rise to estimation bias. Estimation of a noise model in the context of FSF is discussed in (Wang, Gawthrop, and Young, 2005) but the theoretical results for FSF in closed loop are not as yet available. However, our experimental results (Section 5) are encouraging in this respect. The purpose of this section is to solve the second problem. The main tool used for this purpose is the following Lemma.

\section{Lemma 1 If}

$$
\begin{aligned}
y_{a}(t) & =e^{-a t} y(t) \\
u_{a}(t) & =e^{-a t} u(t) \\
g_{a}(t) & =e^{-a t} g(t) \\
y(t) & =g(t) \star u(t)
\end{aligned}
$$

where $\star$ is the convolution operator and the Laplace transforms of $y(t), u(t), g_{a}(t) y_{a}(t), u_{a}(t)$ and $g_{a}(t)$ are $Y(s), U(s)$, $G(s), Y_{a}(s), U_{a}(s)$ and $G_{a}(s)$ respectively, then:

$$
\begin{aligned}
Y(s) & =G(s) U(s) \\
Y_{a}(s) & =G_{a}(s) U_{a}(s) \\
G_{a}(s) & =G(s+a)
\end{aligned}
$$

PROOF. See any Engineering Mathematics text, for example, Kreyszig (1993); in particular, (20) follows from the so-called $s$-shifting theorem.

This idea has been used before in the context of optimal control by Anderson and Moore (1971), but here it is applied to the identification problem. It follows directly from Lemma 1 that if the (possibly unstable) system $G(s)$ (relating $u(t)$ to $y(t))$ has all poles $p_{i}$ such that $\operatorname{Re}\left(p_{i}\right)<a$, the system $G(s+a)$ is stable and relates the exponentially windowed signals (relating $u_{a}(t)$ and $y_{a}(t)$ ). Figure 2 (a) shows the basic scheme for generating the exponentially windowed data. The identification procedure of Section 2.1 can then be used for unstable systems (where $\operatorname{Re}\left(p_{i}\right)<a$ ) but where $y_{i}=y_{a}(i \Delta)$, $u_{i}=u_{a}(i \Delta)$, and the system identified is stable. However, the exponential window effectively discards data for large values of $t$. To overcome this, the standard technique of exponentially weighted least squares (Ljung, 1999) may be used; (7) is replaced by:

$$
J_{b}(M, \hat{\theta})=\sum_{i=0}^{M}\left|e^{b i \Delta} e_{i}\right|^{2}
$$

Using $b=a$ counteracts the effect of the exponential window $e^{-a t}$ and thus all of the data is used. The modified approach for unstable systems is summarised in Figure 2(b).

The use of exponential weighting is prone to numerical problems; numerical rounding can cause small numbers to be treated as zero. For example, it is better to use $e^{(b-a) t}$ rather than $e^{b t} e^{-a t}$ in calculations even though they theoretically have the same value. Hence this method should be used with caution and the minimum appropriate value of $a$ should be used.

\section{Physical Parameter Estimation}

Having compressed the data as in Section 2.1, the impulse response $\hat{g}(t)$ of the empirical system can be obtained from the estimated parameters $\hat{\theta}$ using (12) and then integrated to give the step response $y_{s}(t)$ (13). The estimation problem is to estimate the unknown physical parameters $\Theta$ from the estimated step response $y_{s}(t)$. One possible approach has been considered previously by one of the authors Gawthrop (2000a,b); details necessary for this paper are briefly discussed in Section 3.1 in the context of stable systems and the modifications necessary for unstable systems are considered in Section 3.2.

\subsection{Stable systems}

It is assumed that a mathematical model of physical system is available parameterised by the physical parameter vector $\Theta$ (which may include initial conditions). Given an estimate $\hat{\Theta}$ of $\Theta$, the model can be simulated with a unit step 
input to give an estimated step response $\hat{y}_{s}(t, \hat{\Theta})$. As discussed by Gawthrop (2000a,b), the model is conveniently represented as a Bond Graph (Gawthrop and Smith, 1996; Karnopp, Margolis, and Rosenberg, 2000); however this is not an essential part of the process.

Within this context, the usual least-squares estimation problem is posed; that is to minimise the cost function $J$ with respect to the vector of unknown physical parameters $\Theta$ where:

$$
J(\hat{\Theta})=\frac{1}{2} \int_{0}^{T} e^{2}(t) d t
$$

where the output error $e$ is defined as

$$
e(t)=\hat{y}_{s}(t, \hat{\Theta})-y_{s}(t, \Theta)
$$

Because (23) is not, in general, linear in the parameter estimate $\hat{\Theta}$, this nonlinear least-squares problem does not admit an explicit solution in general; instead, numerical techniques must be used. Each iteration of such an algorithm requires evaluation of the function $J$ for the current estimate $\hat{\Theta}$ and thus a simulation of the system for that value of $\hat{\Theta}$. Thus each iteration is computationally expensive and therefore an efficient algorithm is desirable. For this reason, the simulation is used to generate not only the estimated output $\hat{y}$ but also the derivatives of $J_{\Theta}(\hat{\Theta})$ with respect to $\Theta$. There are many optimisation algorithms available; the Levenberg-Marquardt algorithm (Fletcher, 1987) is used here. This class of algorithms are known to be robust and, as they make use of the sensitivity functions, are more efficient that methods which do not.

\subsection{Unstable systems}

In the case of unstable systems, the estimated system step response corresponds to the stable system $G(s+a)$. Hence, for the purposes of identification, the physical model must be modified in the same way. Because it is a physical system, this can be looked at from various perspectives:

Analogue computers replace all integrators $\frac{1}{s}$ by firstorder lags $\frac{1}{s+a}$;

Mechanical systems add an appropriate damping element to each mass and spring component;

Electrical systems add an appropriate resistive element to each inductive and capacitive component;

The method of Section 3.1 is then applied using the step response of the stable system $G(s+a)$ in conjunction with the stable modified physical system.

\section{Frequency domain data}

Both stages of the estimation procedure described in Sections 2 and 3 use time-domain data. However, as indicated in Figure 3, either of these two stages can use frequencydomain data instead. Ultimately this is a question of taste

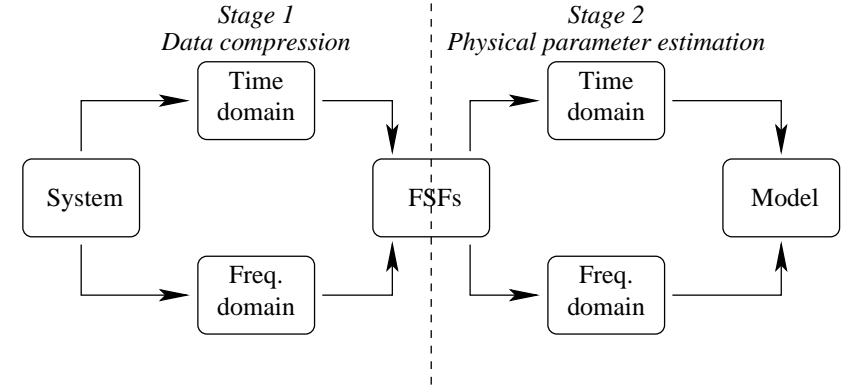

Fig. 3. Estimation data flows

or convenience; thus, for example, the raw data may have been collected using a spectrum analyser. This section discusses briefly the frequency-domain equivalents of the timedomain algorithms reported in Sections 2 and 3. Assume that $M$ output and input frequency-domain data points $Y\left(j \omega_{i}\right)$ and $U\left(j \omega_{i}\right)$ and have been collected at uniformly-spaced frequencies $\omega_{i}=i \Delta_{\omega}, 0 \leq i \leq M$. It is convenient to extend this to a double-sided spectrum $-M \leq i \leq M$ by noting that $Y\left(-j \omega_{i}\right)=Y^{\star}\left(j \omega_{i}\right)$ and $U\left(-j \omega_{i}\right)=U^{\star}\left(j \omega_{i}\right)$ where $\star$ denotes the complex conjugate. The frequency-domain equivalent of (6) is:

$$
Y\left(j \omega_{i}\right)=\theta^{T} F\left(j \omega_{i}\right) U\left(j \omega_{i}\right)
$$

where the convolution of (6) has been replaced by multiplication. Redefining:

$$
\begin{aligned}
& Y_{M}=\left(Y\left(-j \omega_{M}\right) Y\left(-j \omega_{M-1}\right) \ldots Y\left(j \omega_{M}\right)\right)^{T} \\
& \phi_{i}=F\left(j \omega_{i}\right) U\left(j \omega_{i}\right) \\
& \Phi_{M}=\left(\begin{array}{llll}
\phi_{-M} & \phi_{-M+1} & \ldots & \phi_{M}
\end{array}\right)^{T}
\end{aligned}
$$

the least squares estimate of $\theta$ is given by (8).

The method for handling unstable systems given in Section 2.2 and Figure 2 can also be converted to the frequency domain. The basic idea is that time domain multiplication maps into frequency domain convolution. Using the notation of Lemma 1 and noting that the Laplace transform of $e^{-a t}$ is $\frac{1}{s+a}$ the frequency domain equivalent of (14) and (15) is:

$$
\begin{aligned}
Y_{a}(j \omega) & =\frac{1}{j \omega+a} * Y(j \omega) \\
U_{a}(j \omega) & =\frac{1}{j \omega+a} * U(j \omega)
\end{aligned}
$$

From the implementation point of view, however, it is quicker to compute $y(t)$ from $Y(j \omega)$ using the inverse FFT, perform the multiplication (14) and compute $Y_{a}(j \omega)$ from the FFT. Section 3 uses the estimated time-domain response generated from the FSF parameter vector $\theta$ and using a quadratic cost based on the error defined in (23). In a similar fashion, the estimated frequency-domain response can 
be generated from the FSF parameter vector $\theta$. In a similar fashion to (23), a frequency-domain error $E(j \omega)$ is defined as

$$
E(j \omega)=\hat{G}(j \omega, \hat{\Theta})-G(j \omega, \Theta)
$$

By making the corresponding modifications to the equations of Section 3, the physical parameter $\Theta$ can be estimated in the frequency domain.

\section{Experimental Results}

The algorithm has been tested on two experimental systems: one electronic (Sections 5.1 and 5.2) and one electromechanical (Section 5.3). The first is an unstable system and the second has two poles at $s=0$. The first stage of the algorithm was encoded in the Matlab-like language GNU-Octave (www.octave.org) (Eaton, 2002); the second part was implemented as part of the bond-graph toolbox MTT (mtt.sf.net) as described elsewhere (Gawthrop, 2000a).

\subsection{Electronic Unstable system: time-domain}

An unstable electronic circuit was built using the LM124 quad operational amplifier, resistors and capacitors to create a system with voltage to voltage transfer function:

$$
G(s)=\frac{k_{1}}{s^{2}-k_{2}}
$$

where $k_{1}=k_{2}=1$. A proportional + derivative (PD) controller of the form:

$$
u=k\left(w-y-\frac{T_{d} s}{1+T_{f} s} y\right)
$$

was implemented using the real-time software developed by Christini and Culianu (2003) for scientific experimentation using hard real-time control. RTLab runs with the realtime Linux kernel RTAI and the analogue/digital interface Comedi (2002). It was implemented on a Dell Latitude C400 laptop computer using a National Instruments DAQCard1200 PCMCIA card for A/D and D/A conversion. The PD controller sample interval was $\Delta=0.01 \mathrm{sec}$, and the parameters were varied throughout the run; the values do not matter as only the input and output data is of interest.

This is an unstable system and so exponential weighting $(a=2)$ is used. Because non-zero $b$ is not yet implemented for the frequency domain approach $b=0$ in this and the following section to allow comparison between the time and frequency domain approaches. As the signal-to-noise ratio is high, this is not a problem in this example. Figures 4(a) and 4(b) show the experimental data and Figures 4(c) and 4(d) show the time and frequency responses generated from the FSF based estimation with parameters shown in Figure 4. The physical system model was modified (Section 3 ) to shift the poles from $s$ to $s+a$. The estimated physical parameter

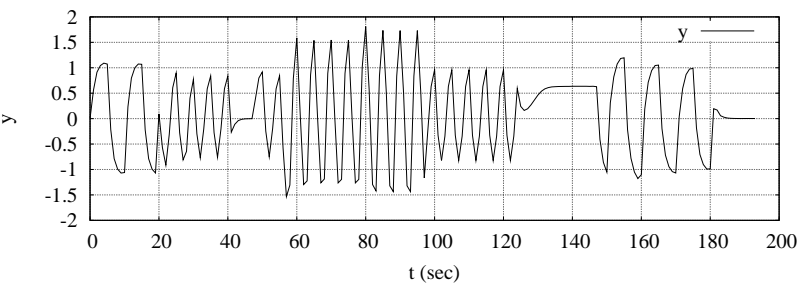

(a) Output $y(t)$

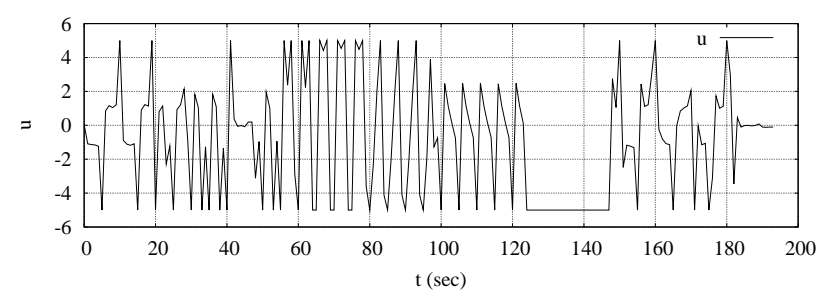

(b) Input $u(t)$

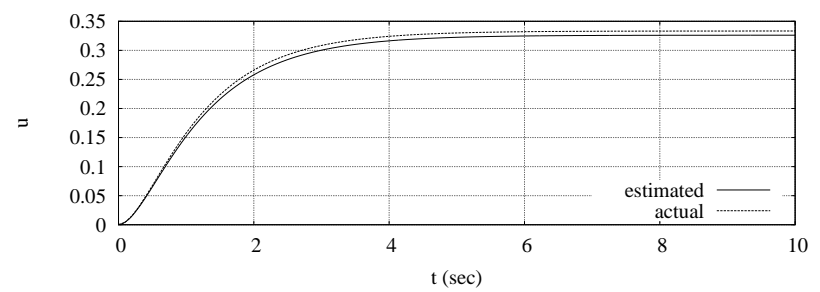

(c) Estimated step response

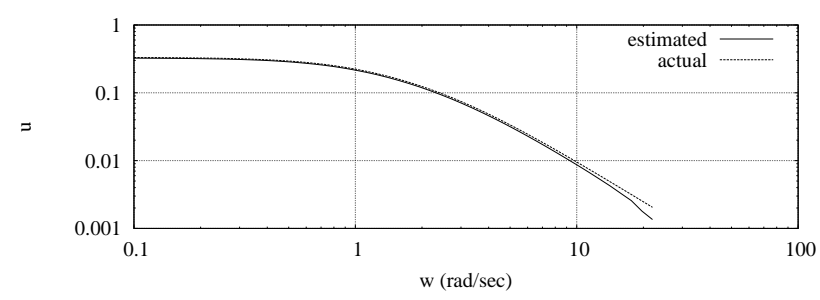

(d) Estimated frequency response

Fig. 4. Experimental Data: Unstable system

$\hat{\Theta}=\left(\begin{array}{ll}\hat{k}_{1} & \hat{k}_{2}\end{array}\right)^{T}$ of (31) converges from a starting value of $\hat{\Theta}=\left(\begin{array}{ll}2.0 & 0.0\end{array}\right)^{T}$ to $\hat{\Theta} \approx\left(\begin{array}{ll}1 & 1\end{array}\right)^{T}$ in about 4 steps.

\subsection{Electronic Unstable system: frequency-domain}

To illustrate the frequency-domain approach of Section 4, the data of Section 5.1 (Figure 4(a)) was Fourier transformed to give the data $Y(j \omega)$ and $U(j \omega)$ shown in Figure 5(a). 


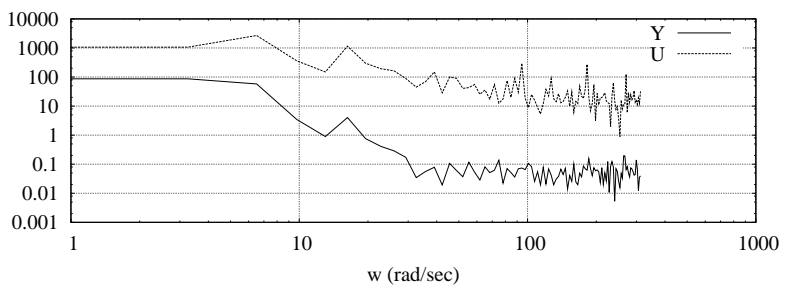

(a) Output $Y(j \omega) \&$ input $U(j \omega)$

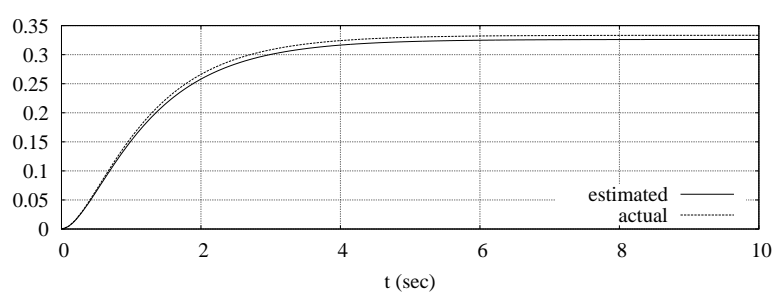

(b) Estimated step and Impulse responses

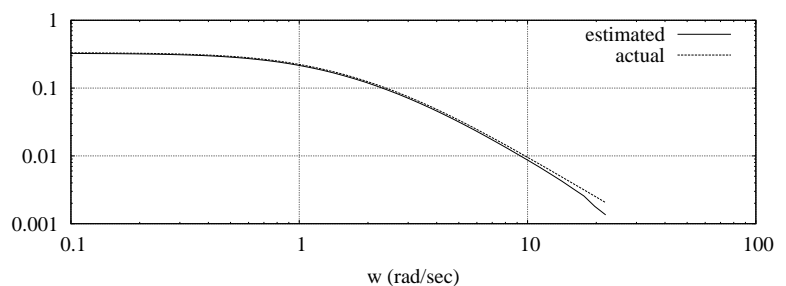

(c) Estimated frequency response

Fig. 5. Experimental Data: Unstable system

Although not the case here, such data would typically be obtained from a commercial spectrum analyser. The FSF parameters were identified from this frequency-domain data using the approach of Section 4 using the same identification parameters as those of Section 5.1; in particular $a=2$ was used to give a stable $\mathrm{G}(\mathrm{s}+\mathrm{a})$. As discussed in the previous section, $b=0$. Unsurprisingly, the step, impulse and frequency responses (Figures 5(b) and 5(c)) are similar to those identified in Section 5.1. The second stage (see Figure 3) was accomplished in the time domain to give results similar to those of Section 5.1.

\subsection{Ball and Beam}

The experimental system was a Quanser Consulting ball and beam experiment (Apkarian, 1995, section 2.2) (with a modified connecting cable and patched inner-loop analogue controller described elsewhere (Gawthrop, 2004)). The experiment can be modelled in detail (Gawthrop, 2004), but for the purposes of this experiment dynamics of the inner

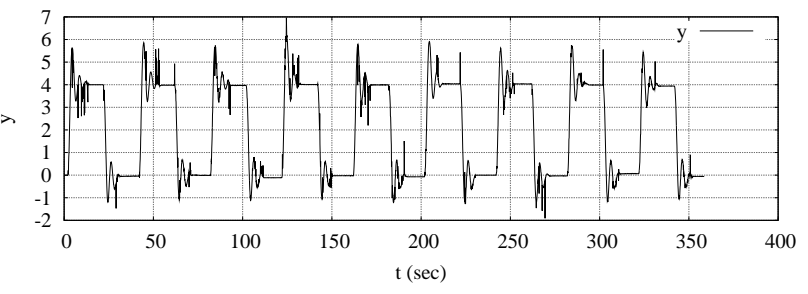

(a) Output $y(t)$

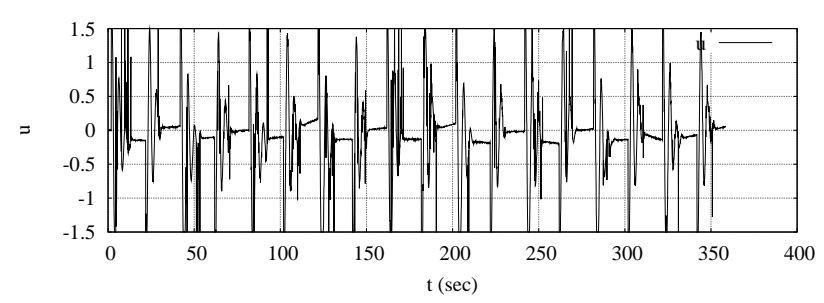

(b) Input $u(t)$

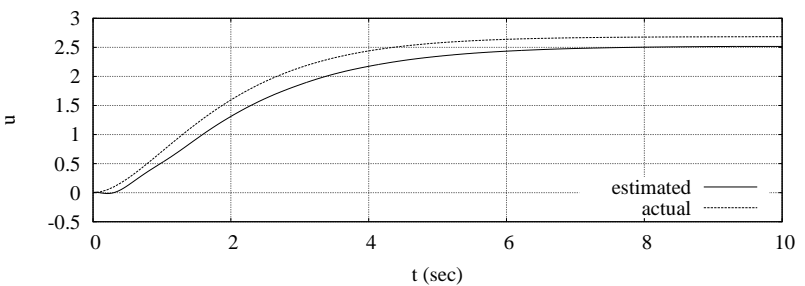

(c) Estimated step response

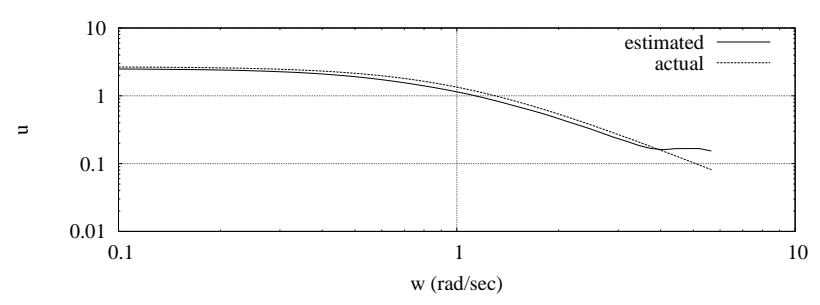

(d) Estimated frequency response

Fig. 6. Experimental Data: Ball-beam system

loop are ignored and thus the system is modelled as:

$$
G(s)=\frac{K}{s^{2}}
$$

where $y$ is the voltage corresponding to the ball position and $u$ the voltage setpoint to the inner-loop controller. This system displays common characteristics of practical mechatronic systems: 
- noisy measurement,

- non-linear stiction on the input and

- neglected high-frequency dynamics.

Figures 6(a) and 6(b) show the ball and beam system under PID control (parameters appear in the Figure caption. Note that the control signal is limited by $-1.5 \leq u \leq 1.5$. This is an unstable system and so exponential weighting $(a=1.0)$ is used. Because the data is noisy a non-zero $b=0.9$ is used here. Figure 6 has the same layout as Figure 4. Figure $6(\mathrm{c}) \&(\mathrm{~d})$ show the estimated step and frequency responses corresponding to

$$
G(s+a)=\frac{K}{(s+a)^{2}}=\frac{K}{(s+1)^{2}}
$$

The physical system model was modified (Section 3) to shift the poles from $s$ to $s+a$. The estimated physical parameter $\hat{\Theta}=\hat{K}$ of (33) converges from a starting value of $\hat{\Theta}=1$ to $\hat{\Theta} \approx 2.5$ in exactly one step; this reflects the fact that the system is linear in the parameter $K$.

\section{Conclusions}

This paper presents a two-step approach for the estimation of the physical parameters of both stable and unstable systems. The central idea is firstly to compress discrete, fast sampled experimental data to a continuous time step response, and secondly to estimate physical parameters within a partially known continuous time transfer function model based on the estimated step response data. An exponential weighting approach is proposed which converts the problem of estimating the physical parameters of an unstable system to an equivalent problem involving a stable system with the same unknown parameters.

The advantages of the proposed approach include improved numerical efficiency and robustness than the more traditional approaches and clear physical insight at each stage. The authors believe that this approach is particularly appropriate when modelling systems for the purposes of continuous-time model-based predictive control; future work will investigate this aspect further.

The approach has been verified using experimental data from an electrical system and an electro-mechanical system.

\section{Acknowledgements}

This work was accomplished whilst the first author was a Visiting Professor at RMIT University, Melbourne supported by the RMIT Professorial fund and the Royal Academy of Engineering (International Travel Grant ITG 03-553).

The real-time software RTLab is part of an Open Source project developed by David Christini and Calin Culianu of Cornell University funded by NSF grant DBI-0096596.
The ball and beam data was generated at the University of New South Wales; the first author acknowledges the facilities provided by Professor V. Solo and the theoretical and practical help provided by Dr David Clements and Chris Lu.

We gratefully acknowledge the important comments on, and contributions to, this paper by the anonymous referees.

\section{References}

K. J. Åström, P. Hagander, and J. Sternby. Zeros of sampled systems. Automatica, 20(1):31-38, 1980.

C. H. An, C. G. Atkeson, and J. M. Hollerbach. Model-based Control of Robot Manipulators. The MIT Press, 1988.

B. D. O. Anderson and J. B. Moore. Linear Optimal Control. Prentice-Hall, Englewood Cliffs, New Jersey, 1971.

Jacob Apkarian. A Comprehensive and Modular Laboratory for Control Systems Design and Implementation. Quanser Consulting, 1995.

R.R. Bitmead and B.D.O. Anderson. Adaptive frequency sampling filters. IEEE Trans. Circuits and Systems, 28: 524-533, 1981.

C. Canudas de Wit. Adaptive control for partially known systems. Elsevier, Amsterdam, 1988.

David Christini and Calin Culianu. RTLab. Online WWW Home Page, 2003. URL: http://www.rtlab.org.

Comedi. Comedi Home Site. Online WWW Home Page, 2002. http://www.comedi.org/.

S. Dasgupta, B. D. O. Anderson, and R. J. Kaye. Output error identification methods for partially known systems. Int. J. Control, 43(1):177-191, 1986.

John W. Eaton. GNU Octave Manual. Network Theory Limited, 2002. ISBN 0-9541617-2-6.

R. Fletcher. Practical Methods of Optimization. 2nd Edition. Wiley, Chichester, 1987.

P. J. Gawthrop. Physical model-based control: A bond graph approach. Journal of the Franklin Institute, 332B(3):285305, 1995.

P. J. Gawthrop, R. W. Jones, and S. A. MacKenzie. Identification of partially-known systems. Automatica, 28(4): 831-836, 1992.

P. J. Gawthrop and L. P. S. Smith. Metamodelling: Bond Graphs and Dynamic Systems. Prentice Hall, Hemel Hempstead, Herts, England., 1996. ISBN 0-13-489824-9.

Peter J. Gawthrop. Estimating physical parameters of nonlinear systems using bond graph models. In Proceedings of the 12th IFAC Symposium on System Identification (SYSID 2000), Santa Barbara, California, USA, June 2000a.

Peter J Gawthrop. Sensitivity bond graphs. Journal of the Franklin Institute, 337(7):907-922, November 2000b.

Peter J Gawthrop. Physically-plausible models for identification. In Proceedings of the 2003 International Conference On Bond Graph Modeling and Simulation (ICBGM'03), Simulation Series, Orlando, Florida, U.S.A., January 2003. Society for Computer Simulation.

Peter J Gawthrop. Bond graph based control using virtual actuators. Proceedings of the Institution of Mechanical 
Engineers Pt. I: Journal of Systems and Control Engineering, 218(4):251-268, September 2004.

Peter J. Gawthrop and Eric Ronco. Estimation and control of mechatronic systems using sensitivity bond graphs. Control Engineering Practice, 8(11):1237-1248, November 2000.

Peter J Gawthrop and Eric Ronco. Predictive pole-placement control with linear models. Automatica, 38(3):421-432, March 2002.

D. C. Karnopp, D. L. Margolis, and R. C. Rosenberg. System Dynamics: A Unified Approach. John Wiley, 1990.

Dean Karnopp, Donald L. Margolis, and Ronald C. Rosenberg. System Dynamics : Modeling and Simulation of Mechatronic Systems. Horizon Publishers and Distributors Inc, 3rd edition, January 2000.

Erwin Kreyszig. Advanced Engineering Mathematics. John Wiley, seventh edition, 1993.

Lennart Ljung. System Identification: Theory for the User. Information and Systems Science. Prentice-Hall, 2nd edition, 1999.

Lennart Ljung and Torkel Glad. Modeling of Dynamic Systems. Prentice Hall, 1994.

D.Q. Mayne, J.B. Rawlings, C.V. Rao, and P.O.M. Scokaert. Constrained model predictive control: Stability and optimality. Automatica, 36(6):789-814, June 2000.

Neil Munro, editor. Symbolic methods in control systems analysis and design. Number 56 in Control engineering series. IEE, Stevenage, UK, 1999.

P. A. J. Nagy and L. Ljung. System identification using bond graphs. In Proceedings of the 1st European Control Conference, pages 2564-2569, Grenoble, France, 1991. Hermes.

B. Ninness and F. Gustafsson. A unifying construction of orthonormal bases for system identification. IEEEAC, 42 (4):515-521, 1997.

James B. Rawlings. Tutorial overview of model predictive control. IEEE Control, 20(3):38-52, June 2000.

B. Wahlberg. System identification using Laguerre models. IEEE Transactions on Automatic Control, 36:551-562, 1991.

L. Wang. Continuous time model predictive control using orthonormal functions. Int. J. Control, 74:1588-1600, 2001.

L. Wang and W. R. Cluett. Frequency-sampling filters: an improved model structure for step-response identification. Automatica, Vol. 33(5):939-944, 1997.

L Wang and W R Cluett. From Plant Data to Process Control. Taylor and Francis, London and New York, 2000.

L. Wang, P.J. Gawthrop, and P.C. Young. Continuous-time system identification of nonparametric models with constraints. In Proceedings of the 16th IFAC World Congress, Prague, 2005.

Liuping Wang, Peter Gawthrop, Charlie Chessari, Tony Podsiadly, and Angus Giles. Indirect approach to continuous time system identification of food extruder. Journal of Process Control, 14(6):603-615, September 2004. 